\title{
Particle-pressure-induced self-filtration in concentrated suspensions
}

\author{
Sandeep D. Kulkarni, ${ }^{1}$ Bloen Metzger, ${ }^{2}$ and Jeffrey F. Morris ${ }^{1}$ \\ ${ }^{1}$ Levich Institute and Chemical Engineering, City College of New York, New York, New York 10031, USA \\ ${ }^{2}$ CNRS Laboratoire IUSTI, Marseille, France
}

(Received 9 November 2009; published 21 July 2010)

\begin{abstract}
Gravity-driven flow of concentrated suspensions (solid volume fraction $\phi>0.50$ ) of non-Brownian spherical particles through a channel contraction at low Reynolds number is studied experimentally. The abrupt change in the flow area at the contraction forms distinct shear-rate regions having different fluid pressures, which are related to the concept of particle pressure. A model involving particle pressure variation coupled to a Darcylike behavior for the fluid captures the phenomenon of "self-filtration," in which the effluent material has lower solid fraction than the input suspension. For $\phi \doteq \phi_{\text {crit }} \approx 0.58$, under added load from a weighted piston, the flow periodically alternates between fast and slow motions.
\end{abstract}

\section{DOI: 10.1103/PhysRevE.82.010402}

\section{INTRODUCTION}

Flows of concentrated suspensions, from mud to ceramic precursor slurries, are commonly encountered in nature and industry. Suspensions range from colloidal dispersions to wet granular flows and thus have rich and varied dynamics. Concentrated colloidal suspension flows, with solid fraction $\phi$ approaching the maximum packing limit, exhibit phenomena including erratic speed fluctuations [1], confinement-induced flow oscillations [2], and "giant" stress fluctuations when the imposed shear rate approaches a transition value [3]. In liquid-immersed granular flows, dilatancy effects are seen for underwater granular avalanches [4], while flow instabilities are observed for inclined-plane flows beyond a critical inclination angle [5]. In this work, we address self-filtration, in which the effluent from a pressure-driven contraction flow has lower solid loading than the upstream material [1]; this phenomenon is similar to behavior previously studied in the context of suspension and paste extrusion [6,7]. The present study of flow of dense noncolloidal suspensions through a contraction elucidates the development of a flow-induced liquid pressure field and its relation to self-filtration in steady and oscillatory flows.

Particle migration is observed in a number of lowReynolds-number suspension flows, including curvilinear [8] and pressure-driven rectilinear flows [9]. The migration has been modeled based on "particle pressure" as driving potential $[10-12]$. The particle pressure $\Pi$ is the mean normal stress exerted by the particle phase $\Pi=-\frac{1}{3}\left[\sum_{11}^{p}+\sum_{22}^{p}+\sum_{33}^{p}\right]$ in a sheared suspension [13]. A compressive particle pressure $\Pi$ quantifies the tendency of the dispersed phase to spread under shear. For this spreading to take place, liquid must replace the particles, and there must be an equal but opposite (and hence, suction) pressure in the suspending liquid $\Pi_{l i q}=-\Pi(\dot{\gamma}, \phi)$; this is deduced as a result of the constraint of material incompressibility $[12,14]$. The relation of diffusion with liquid pressure in solid-liquid systems is a broadly relevant topic, e.g., for the ultrafiltration process, where theory of the suspending liquid pressure has established equivalency of Fick's and Darcy's laws [15]. Measurements at a porous boundary show evidence of opposing normal stresses in the two phases of sheared suspensions [16]. A clear demonstration is provided by measurement of the solvent pressure in a sheared suspension by a method analogous
PACS number(s): $83.80 . \mathrm{Hj}, 82.70 . \mathrm{Kj}$

to measurement of osmotic pressure in quiescent solutions across a semipermeable membrane [14].

In this work, we study a contraction geometry, in which distinct shear-rate regions form and are found to have significantly different solvent phase suction pressures $\Pi_{l i q}$. In addition, at large $\phi$, we observe a reduction in suspension concentration downstream of the contraction relative to the upstream. This dilation or self-filtration and its relation to the flow-induced liquid pressures are studied for steady and oscillatory flow conditions.

\section{EXPERIMENTS}

The suspensions are composed of spherical polystyrene particles (380-500 $\mu \mathrm{m}$ in diameter; Maxi-Blast Inc.) and UCON 50-HB-660 (Dow Chemical), a Newtonian liquid with viscosity $\eta_{f}=0.3 \mathrm{~Pa}$ s and density $1.05 \mathrm{~g} \mathrm{~cm}^{-3}$ at $23^{\circ}$, very close to that of polystyrene. Inertia is negligible as $R e_{p}=\rho U a / \eta_{f} \ll 1$, where $U$ is the average flow speed, $a$ is the sphere radius, and $\rho$ and $\eta_{f}$ are the fluid density and viscosity, respectively. Flows of two more viscous liquids without particles, mixtures of UCON 75-H-90000 and 75-H1400 (both of density $1.09 \mathrm{~g} \mathrm{~cm}^{-3}$ ) having viscosities $36.5 \mathrm{~Pa} \mathrm{~s}$ (liquid $X$ ) and $7 \mathrm{~Pa} \mathrm{~s}$ (liquid $Y$ ) at $23^{\circ}$, are also studied.

The flow passes through a contraction in a vertical channel constructed of acrylic, with geometry shown in Fig. 1. The length, width $(W)$ and depth of the channel are 20, 2.5, and $2 \mathrm{~cm}$, respectively. The contraction is formed by symmetrically inserting two rectangular blocks of length $4 \mathrm{~cm}$, depth $2 \mathrm{~cm}$ (equaling the channel depth), and of width yielding the ratio of the contraction channel width to that of original channel $w / W=1 / 6$ (Fig. 1). A charge coupled device (CCD) camera (Basler A1021) images the flow along an axis orthogonal to the channel wall.

The suspension is poured into the channel to a level 15 $\mathrm{cm}$ above the contraction, and then a bottom plate restricting outflow is removed. The effluent material collected at the exit as the suspension column height drops about $7 \mathrm{~cm}$ is weighed; the particles are recovered by thorough washing and drying, then weighed separately to obtain the outlet concentration. Unless mentioned, the upper surface of the suspension is open to the atmosphere. Experiments with addi- 


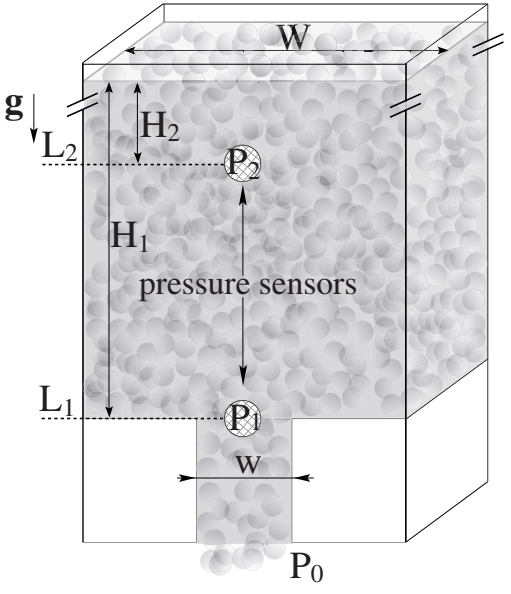

FIG. 1. Sketch of the experiment.

tional driving force use a closely fitted weighted piston placed on the upper surface of the mixture.

The liquid pressures $P_{1}$ and $P_{2}$ in flowing systems are measured by differential transducers (Omega; \pm 1 psi) attached across holes in the channel wall at the contraction (level $L_{1}$ ) and $5 \mathrm{~cm}$ above (level $L_{2}$ ); reported pressures are thus relative to atmospheric. We confirm experimentally that the extent of significant nonaxial flow above the contraction level is $\approx 3 w: L_{2}$, well above this zone, is in a unidirectional flow region. For suspension flows, liquid pressure alone rather than that exerted by both particles and fluid is measured by placing a mesh across each hole to retain the particles, as previously shown [14].

Figure 2 shows $P_{1}$ vs $H_{1}$ and $P_{2}$ vs $H_{2}$ for flow of different materials through the contraction, where $H_{1}$ and $H_{2}$ are heights of liquid (or suspension) column at respective measurement levels as shown in Fig. 1. For the pure liquid $(\phi=0)$ flow, the pressures $P_{1}$ and $P_{2}$ are slightly below the corresponding static heads $\rho g H_{1}$ and $\rho g H_{2}$ (indicated by solid lines), as a result of viscous pressure losses accounted as $\Delta P_{1}=\rho g H_{1}-P_{1}$, as labeled in Fig. 2(a), and $\Delta P_{2}=\rho g H_{2}-P_{2}$. Figure 2 shows that, for pure liquids $X$ and $Y$ differing in viscosity by a factor of over five, the pressure (and hence $\Delta P$ ) dependence on fluid-column height is identical. In contrast, suspension flows are characterized by reduction in liquid pressures, differing with volume fraction; this reduction for a suspension of $\phi=0.5$ is labeled as $\Pi_{1}$ in Fig. 2(a) to indicate the relationship to particle pressure which we develop later.

We note that the equivalence of pressure dependence for different viscosity pure liquids is a consequence of hydrodynamic linearity under the Stokes-flow conditions. The viscous loss $\Delta P_{1}$ (or $\Delta P_{2}$ ) is proportional to the shear stress $\eta_{s} \dot{\gamma}$. The shear rate may be written as $\dot{\gamma}=K(\mathbf{x})\left(\rho g H_{1} / \eta_{s}\right)$, where $K(\mathbf{x})$ accounts for spatial variation in shear rate due to the contraction; the liquid column head $\rho g H_{1}$ above the contraction is the driving force for flow, and the resistance to flow scales with $\eta_{s}$. Thus $\Delta P_{1} \sim \eta_{s} \dot{\gamma} \sim \rho g H_{1}$, confirming independence of viscous losses on material viscosity. The experimental data for both liquids yield $\Delta P_{1}=0.16 \rho g H_{1}$ and $\Delta P_{2}=0.03 \rho g H_{1}$. We have confirmed that in the absence of flow, the liquid pressure is equivalent to the static fluid head.
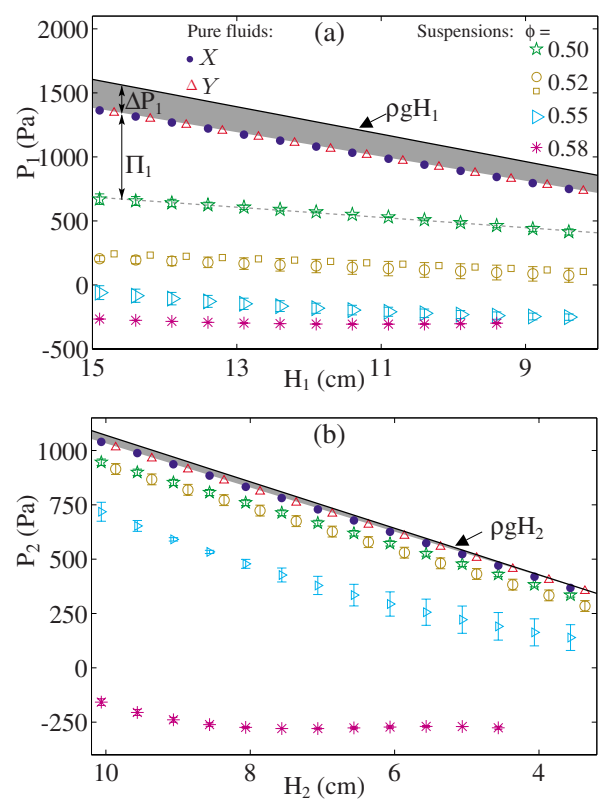

FIG. 2. (Color online) Liquid pressure of flowing systems measured (a) at $L_{1}$ in the beginning of the contraction; (b) at $L_{2}, 5 \mathrm{~cm}$ vertically above $L_{1}$, plotted against fluid-column height at each measurement level. The legend in (b) applies also to (a). Viscosities are for liquid $X 36.5 \mathrm{~Pa}$ s and for liquid $Y 7 \mathrm{~Pa}$ s; For suspensions, the suspending fluid has $\eta_{f}=0.3 \mathrm{~Pa}$ s, except for one set of data at $P_{1}$ for $\phi=0.52$, where $\eta_{f}=0.9 \mathrm{~Pa} \mathrm{~s}$ (open squares).

Also, when the particle flow is restricted by a mesh at the bottom yielding Darcy flow through a column of particles, we find $d P / d z \approx 50 \mathrm{~Pa} / \mathrm{cm}$ for $\phi \geq 0.55$.

We now assess the liquid pressure for flow of suspensions at solid fraction $\phi=0.50,0.52,0.55$, and 0.58 , with $\eta_{f}=0.30 \mathrm{~Pa} \mathrm{~s}$ (in one case $\eta_{f}=0.9 \mathrm{~Pa} \mathrm{~s}$ ). The suspension effective viscosity satisfies $\eta_{s}=\eta_{s}^{*}(\phi) \eta_{f}$, where $\eta_{s}^{*} \sim\left(1-\phi / \phi_{\max }\right)^{-2}$, with $\phi_{\max } \approx 0.6$ the maximum packing fraction. Recall that the liquid pressure alone is obtained by placing screens across holes at $L_{1}$ and $L_{2}[14]$ and that the pressure drop due to viscous losses is independent of the viscosity $\eta_{s}$ and depends (for fixed density) only on fluidcolumn height $H_{1}$. Yet Fig. 2 shows that the measured liquid pressure values for a suspension decrease relative to those found for the pure liquid flows, and the decrease becomes more pronounced with increasing $\phi$. The striking conclusion is that the additional decrease in liquid-phase pressures $P_{1}$ and $P_{2}$ observed for suspensions must be due to another physical process unique to a two-phase flow. As noted above, experiments [14] and simulations [12] have shown that uniform shearing in suspensions generates suction pressure in the suspending liquid $\Pi_{l i q}=-\Pi(\dot{\gamma}, \phi)$, which is accounted here as

$$
P_{1}=\rho g H_{1}-\Delta P_{1}-\Pi_{1}, \quad P_{2}=\rho g H_{2}-\Delta P_{2}-\Pi_{2} .
$$

Because $\Delta P_{1}$ and $\Delta P_{2}$ were previously evaluated and independent of viscosity, we are able to determine $\Pi_{1}$ and $\Pi_{2}$ separately as a function of fluid-column height.

The measurement of self-filtration, where effluent concentration $\phi_{\text {out }}^{m}$ is below the input $\phi_{i n}$, is shown in Fig. 3. For 


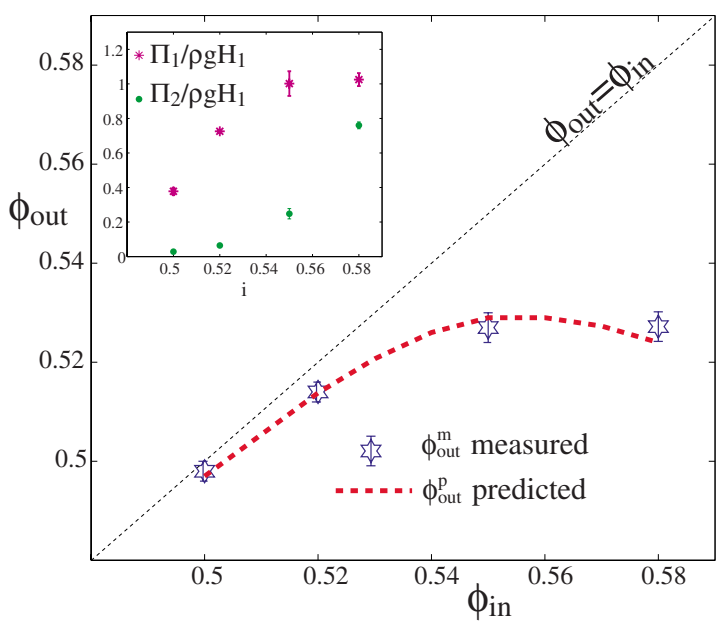

FIG. 3. (Color online) Comparison of the measured and predicted outlet particle volume fractions, $\phi_{\text {out }}^{m}$ and $\phi_{\text {out }}^{p}$ for contraction geometry $w / W=1 / 6$; the inset shows $\Pi_{1} / \rho g H_{1}$ and $\Pi_{2} / \rho g H_{1}$ plotted against input particle concentration $\phi_{i n}$.

$\phi_{\text {in }} \geq 0.55$, we find $\phi_{\text {out }}^{m} \approx 0.528 \pm 0.003$. Also, $\phi_{\text {out }}^{m}$ remains unchanged as the suspension column drops from $H_{1}=15$ to 8 $\mathrm{cm}$. As a result of mass conservation and dilution of the effluent, the top of the column would become dry (as we observed in early experiments), unless a layer of a few $\mathrm{mm}$ depth of pure liquid is added on top of the suspension, and we follow this practice. Similar filtration behavior for colloidal systems was observed by [1], where it was suggested that colloidal jamming or flow instabilities are necessary to induce self-filtration. On the contrary, we observe this phenomena under steady flow.

\section{MODEL}

We now propose a mechanistic model for self-filtration invoking particle pressure and check its consistency with our experimental results. Constitutive modeling [10] and simulation at uniform shear and $\phi[11,12]$ have shown that particle pressure may be expressed as $\Pi=\eta_{n} \dot{\gamma}$ for non-Brownian suspensions, where $\eta_{n}=\eta_{n}^{*}(\phi) \eta_{f}$ is the suspension "normal stress viscosity" modeled as $\eta_{n}^{*} \sim 0.75\left(\phi / \phi_{\max }\right)^{2}\left(1-\phi / \phi_{\max }\right)^{-2}$. Combining the local rheology $\Pi \sim \eta_{n} \dot{\gamma}$ with dependence of the shear rate on driving head, $\dot{\gamma}=K(\mathbf{x})\left(\rho g H_{1} / \eta_{s}\right)$,

$$
\frac{\Pi}{\rho g H_{1}} \approx K(\mathbf{x}) q(\phi),
$$

where $q(\phi)=\eta_{n} / \eta_{s}$ is the ratio of normal to shear stress (or inverse of friction coefficient in granular rheology) which increases with $\phi$ and is independent of $\eta_{f}[10,17]$. This formulation leads to the remarkable implication that for a fixed configuration and $\phi$ in gravity-driven flow, particle pressure is independent of the suspending liquid viscosity. We confirm this experimentally, as illustrated by the two identical curves for $P_{1}$ (within error bar) in Fig. 2(a) at $\phi=0.52$, for $\eta_{f}=0.3$ or $0.9 \mathrm{~Pa} \mathrm{~s}$. A second implication is that at a given point in the channel the ratio $\Pi / \rho g H_{1}$ is independent of $H_{1}$ and increases sharply with $\phi$. At the positions of the pressure sensors, we plot $\Pi_{1} / \rho g H_{1}$ and $\Pi_{2} / \rho g H_{1}$ averaged over $8<H_{1}<15 \mathrm{~cm}$ for experiments with different $\phi_{\text {in }}$ (Fig. 3 inset). The small error bars confirm independence of $H_{1}$ and no considerable change in local $\phi$ over any experiment. The sharp rise in $\Pi_{2} / \rho g H_{1}$ with $\phi_{i n}$ is expected, but given the constitutive modeling, the flattening of $\Pi_{1} / \rho g H_{1}$ suggests that $\phi$ at the contraction is reduced relative to $\phi_{i n}$ and is essentially unchanged for $\phi_{i n} \geq 0.55$. This interpretation of dilation at the contraction agrees with flattening of measured outlet concentration $\phi_{\text {out }}^{m}$ for $\phi_{\text {in }} \geq 0.55$.

To address the basis of the observed dilation, we now assume that the fluid pressure variation between contraction and bulk regions induces flow of liquid relative to particles, similar to Darcy flow, sucking liquid toward the contraction. This suction force can be estimated as $\left[\Pi_{1}-\Pi_{2}\right]_{\phi=\phi_{i n}}$. A direct measure is difficult, as $\Pi_{1}$ is affected by dilation at the contraction. We reformulate the suction force in terms of $\Pi_{2}$ using Eq. (2),

$$
\left[\Pi_{1}-\Pi_{2}\right]_{\phi=\phi_{i n}}=\left(K_{1} / K_{2}-1\right) \Pi_{2},
$$

where we recall that $K$ represents the spatial dependence of the shear rate. The flow of liquid relative to particles $\Delta V=V_{l}-V_{p}$ is stated as

$$
\frac{\Delta V}{V_{p}} \sim \frac{\Pi_{2} / \eta_{s}}{\rho g H_{1} / \eta_{s}} \sim \frac{\Pi_{2}}{\rho g H_{1}} .
$$

As $\Pi_{2} / \rho g H_{1}$ is independent of $H_{1}$, Eq. (4) implies steady dilution, consistent with the experimental observation. Writing a conservation equation for the particles and incorporating Eq. (4) yield

$$
\phi_{\text {out }}=\frac{\phi_{\text {in }} V_{p}}{\phi_{\text {in }} V_{p}+\left(1-\phi_{\text {in }}\right) V_{l}}=\frac{\phi_{\text {in }}}{1+A \frac{\Pi_{2}}{\rho g H_{1}}\left(1-\phi_{\text {in }}\right)} .
$$

Using the experimental measurement of $\Pi_{2} / \rho g H_{1}$ and $\phi_{\text {out }}$ from Fig. 3, we obtain the dimensionless $A=0.34 \pm 0.04$. With this value of $A$ and a quadratic interpolation of $\Pi_{2} / \rho g H_{1}$ data in Fig. 3 inset, the prediction of the effluent concentration is in satisfactory agreement with the experimental data (Fig. 3); the downward curvature is an artifact of the quadratic fit.

Considering the spatial dependence of the shear rate $\dot{\gamma}$ $=K(\mathbf{x})\left(\rho g H_{1} / \eta_{s}\right)$, one would expect $K_{1} / K_{2}=6$ in the present geometry where the ratio of the contraction channel $w / W$ $=1 / 6$. However, for $\phi=0.50$ and 0.52 , where the observed dilation is small, Eq. (2) suggests $K_{1} / K_{2} \approx \Pi_{1} / \Pi_{2} \approx 13$. This larger value shows that $K_{1} / K_{2}$ is influenced both by the variation in shear rate and change in flow type from pure shear flow to extensional flow at the contraction. We also conducted experiments with smaller particles (150-250 $\mu \mathrm{m}$ in diameter) at $\phi=0.58$. The pressure responses $P_{1}$ and $P_{2}$ as well as the effluent concentration $\left(\phi_{\text {out }}^{m} \approx 0.53\right)$ remains unaffected by the particle size for the studied range.

\section{OSCILLATORY FLOW UNDER ADDED LOAD}

We now consider flow under additional load supplied by a $100 \mathrm{~g}$ piston added to the top of the mixture. The weight is 


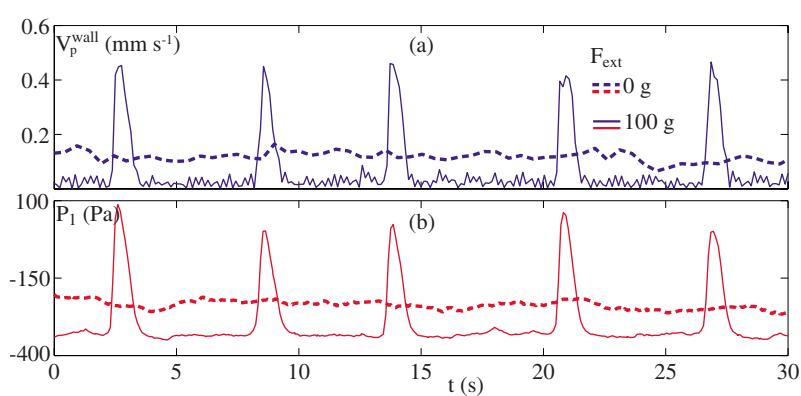

FIG. 4. (Color online) (a) Velocity, $V$, and (b) liquid pressure, $P_{1}$, at the contraction for $\phi=0.58$ and $w / W=1 / 6$ : dashed lines correspond to no external force; solid lines correspond to a weighted piston of $100 \mathrm{~g}$ load.

equivalent to $2 \mathrm{kPa}$ additional pressure. Images are captured using a high magnification lens (Sigma DG HSM) with wide field of view $(1.5 \mathrm{~cm} \times 2 \mathrm{~cm})$ focused on the contraction, illuminated by white light. Particle tracking velocimetry (PTV) is used to obtain the average particle speed along the wall. The bulk flow determined from the decline of the suspension column is 4-5 times the observed near wall motion for both steady and oscillatory flows. In the contraction of $w / W=1 / 6$, at $\phi=\phi_{\text {crit }} \approx 0.58$ with the weighted piston, PTV shows that the flow alternates between fast and slow motions with a surprising periodicity as shown in Fig. 4 (see also the movie available with the online version of the paper [18]). The oscillations are absent below a particular threshold load as seen in earlier work [2], but the oscillation frequency here decreases with added load, contrary to that study. Robustness of periodic flow to changes in geometry, suspending liquid viscosity and piston weight has been established but to be brief we focus on $\phi=0.58, w / W=1 / 6$ and added piston load of $100 \mathrm{~g}$. Under this condition, the measured effluent concentration is $\phi_{\text {out }}^{m}=0.527 \pm 0.003$ with the stated variance based on multiple experimental trials. Figure 4 shows that the particle speed in slow motion of the alternating flow is lower than with no external force. This suggests the weighted piston makes contact with particles at the upper surface, activating a granular network which supports most of the piston weight. Slow creep of the deforming granular network causes a decrease in liquid pressure $P_{1}$. When the network abruptly breaks, as indicated by the faster motion, the internal stress in the particle phase is released and the liquid pressure goes up simultaneously (Fig. 4). Thus, dilational motion near the contraction in the slow regime ultimately leads to breakage of the granular network. As the diluted batch passes through the contraction, the granular network is reestablished and begins again to creep, thus leading to periodic flow variation. Similar correlation between flow speed and pore pressure is observed in avalanching flows [4]. As a final point, note that the threshold load needed to induce oscillations is approximately $50 \mathrm{~g}$ for typical particles (380-500 $\mu \mathrm{m}$ in diameter) and $500 \mathrm{~g}$ for smaller particles (150-250 $\mu \mathrm{m}$ in diameter), suggesting the influence of channel size/particle ratio.

We conclude that in concentrated suspension flow in a contraction geometry, the change in shear rate at the contraction induces a spatially varying pressure field in the liquid phase, with the pressure reduced well below the value expected due to viscous losses. In the same flow, we observe self-filtration with a reduced outlet solid concentration relative to the imposed inlet concentration. We have related the reduction in the liquid pressure field to particle pressure and proposed a model which rationalizes the self-filtration phenomenon. When driving the system with a weighted piston at $\phi_{\text {crit }} \approx 0.58$, the flow shows consistent periodic oscillations where fast and slow motion of the suspension correspond, respectively, to low and high internal stress states of the particle phase. The same type of coupling involving dilation and failure of the solid network seems responsible for this striking phenomenon. Note that the liquid pressure in the porous medium state of the mixture is equivalent to a pore pressure in granular and soil mechanics [19]. We conclude that the concept of pore pressure is directly related to shear-induced particle pressure, $\Pi$, in suspensions, which has been established both as a nonequilibrium continuation of osmotic pressure [12] and a general drive toward particle phase dilation [14].
[1] M. D. Haw, Phys. Rev. Lett. 92, 185506 (2004).

[2] L. Isa, R. Besseling, A. N. Morozov, and W. C. K. Poon, Phys. Rev. Lett. 102, 058302 (2009).

[3] D. Lootens, H. Van Damme, and P. Hebraud, Phys. Rev. Lett. 90, 178301 (2003).

[4] M. Pailha and O. Pouliquen, J. Fluid Mech. 633, 115 (2009).

[5] C. Cassar, M. Nicolas, and O. Pouliquen, Phys. Fluids 17, 103301 (2005).

[6] P. Yaras, D. M. Kalyon, and U. Yilmazer, Rheol Acta. 33, 48 (1994).

[7] S. L. Rough, J. Bridgwater, and D. I. Wilson, Int. J. Pharm. 204, 117 (2000).

[8] D. Leighton and A. Acrivos, J. Fluid Mech. 181, 415 (1987).

[9] C. J. Koh, P. Hookham, and L. G. Leal, J. Fluid Mech. 266, 1 (1994).

[10] J. F. Morris and F. Boulay, J. Rheol. 43, 1213 (1999).

[11] A. Sierou and J. F. Brady, J. Rheol. 46, 1031 (2002).
[12] Y. Yurkovetsky and J. F. Morris, J. Rheol. 52, 141 (2008).

[13] D. J. Jeffrey, J. F. Morris, and J. F. Brady, Phys. Fluids A 5, 2317 (1993).

[14] A. Deboeuf, G. Gauthier, J. Martin, Y. Yurkovetsky, and J. F. Morris, Phys. Rev. Lett. 102, 108301 (2009).

[15] S. S. L. Peppin, J. A. W. Elliott, and M. G. Worster, Phys. Fluids 17, 053301 (2005).

[16] D. Prasad and H. K. Kytomaa, Int. J. Multiphase Flow 21, 775 (1995).

[17] I. E. Zarraga, D. A. Hill, and D. T. Leighton, J. Rheol. 44, 185 (2000).

[18] See supplementary material at http://link.aps.org/supplemental/ 10.1103/PhysRevE.82.010402 for a movie showing the periodic flow, $\phi=0.58, w / W=1 / 6, F_{\text {ext }}=100 \mathrm{~g}$.

[19] R. M. Iverson, M. E. Reid, and R. G. LaHusen, Annu. Rev. Earth Planet. Sci. 25, 85 (1997). 